\title{
AUTOMATIC DEPENDENT SURVEILLANCE - BROADCAST / COCKPIT DISPLAY OF TRAFFIC INFORMATION: PILOT USE OF ELECTRONIC VS PAPER MAP DISPLAYS DURING AIRCRAFT NAVIGATION ON THE AIRPORT SURFACE
}

O. Veronika Prinzo, Ph.D.

Federal Aviation Administration, Office of Aerospace Medicine, Civil Aerospace Medical Institute, AAM-510, 6500 S MacArthur Bvd, Oklahoma City, OK 73179

\begin{abstract}
The Federal Aviation Administration is making a concerted effort to reduce runway incursions. A 5-day operational evaluation, conducted in October 2000 , assessed pilot use of varying types of CDTI devices. Structured and unstructured taxi routes examined how well pilots navigated their aircraft using an electronic surface-map display (north-up, track-up) or a paper surface map. An analysis of 15 hours of communication data was performed to determine how the use of these displays might aid situation awareness and influence operational communications. A Type-of-Route $\mathrm{x}$ Type-of-Map ANOVA revealed more problems occurred and more messages were exchanged for structured taxi routes. A statistically significant interaction indicated most problems occurred for the north-up map during structured taxi routes and the number of problems encountered was comparable for the other maps when pilots navigated along unstructured taxi routes. Avionics developers may want to reconsider north-up surface moving map displays airport surface navigation tasks.
\end{abstract}

Key words: CDTI, ADS-B, moving map display

\section{INTRODUCTION}

Recreational and professional pilots form a diverse population of aviators who vary in piloting skills, experience with airport operations, and familiarity with the surface geography of their departure and destination airports. At one time or another, they - like all of us - make mistakes. 
Sometimes, adverse weather or poor visibility add complexity and contribute to human error. The more serious mistakes can result in runway incursions, surface incidents, near-collision ground incidents, and fatal runway collisions.

In its special-investigation report entitled Runway Incursions at Controlled Airports in the United States (May 6, 1986), the National Transportation Safety Board (NTSB) noted a significant increase in collision ground incidents ${ }^{13}$. That report included several new safety recommendations to reduce the frequency of runway incursions. Some of these recommendations remained open when, on January 18, 1990, a fatal runway collision involving a Boeing 727 and a Beechcraft King Air A100 occurred at Atlanta, Georgia. As a result, the NTSB placed airport runway incursions on its "1990 Most Wanted Transportation Safety Improvements List," where it still remains.

The FAA is working diligently to address NTSB Safety Recommendation A-00-66 (NTSB, 2000): “... require, at all airports with scheduled passenger service, a ground movement safety system that will prevent runway incursions; the system should provide a direct warning capability to flight crews. In addition, the FAA should demonstrate through computer simulations or other means that the system will, in fact, prevent incursions." ${ }^{14}$ A critical component of Safety Recommendation A-00-66 is that runway incursion prevention technologies should "provide a direct warning capability to flight crews."

In 2000 and again in 2002, the FAA's Office of Runway Safety made a concerted effort to reduce runway incursions. Several technologies that are being developed will provide a direct alerting capability to flight crews include ground markers, addressable signs, and surface moving maps. Under the Safe Flight 21 Program, contracts were awarded for avionics development and demonstration that included a surface moving-map capability. This capability was demonstrated (along with several others) in October 2000 during an operational evaluation of the automatic dependent surveillance broadcast (ADS-B) and cockpit display of traffic information (CDTI).

The FAA's intent in undertaking operational evaluation activities is to refine, standardize, and certify a set of tools that airports can acquire to

${ }^{13}$ In 1987, the FAA Administrator approved the definition of the term "runway incursion" as "any occurrence at an airport involving an aircraft, vehicle, person, or object on the ground that creates a collision hazard or results in loss of separation with an aircraft taking off, intending to take off, landing, or intending to land." This definition was clarified in 1996 to refer only to airports with operating control towers (Order 7050.12002 ).

${ }^{14}$ Letter of recommendation dated July 6,2000, to the FAA addressing runway incursions. 
address their specific runway safety issues. The stated purposes of the operational evaluation were to develop and evaluate specific ADS-B air-air and air-ground applications, evaluate controller use of ADS-B, and demonstrate ADS-B technology. It also provided an opportunity to collect field data that could be used to guide the development of the ADS-B airport surface movement applications. These applications would improve surface surveillance and navigation through enhancements to airport surface situation awareness.

One goal associated with the airport surface situational awareness application was to enhance safety and mitigate occasions for runway incursion by providing pilots with tools that graphically display the proximate location of other surface aircraft and vehicles. Another was to enhance positional awareness by providing them with tools that displayed real-time information to supplement out-the window speed, direction, and position information.

To evaluate how ADS-B and surface-map information could be used to aid pilot situation awareness, very specific and complex taxi routes were created to examine how well flight crews navigated their aircraft along the assigned taxi routes using either an electronic surface-map display or a paper surface map. During the five-day event, objective (air traffic control voice tapes and radar data) and subjective data (surveys, questionnaires, jump-seat observer reports, small-group interviews) were collected. This report provides a general description of the communication findings.

\section{METHOD}

\subsection{Participants}

Twenty-five paid pilot volunteers flew 16 different aircraft. Two controllers and a coordinator (also volunteers) provided local- and groundcontrol services. They were on a temporary detail during training and on a regular schedule during the evaluation.

\subsection{Materials}

\subsubsection{Experimental Structured Taxi Routes and Taxi-Route Cards}

The experimentally constructed taxi routes (structured taxi routes) described the routes for pilots to navigate a defined course segment to or from the assigned runway. Pilots received individual uniquely labeled cards with these "canned" taxi routes presented in text format. Each card had a 
named taxi route associated with it (e.g., CUPS1, FBO1, ANG1) that provided very specific, and often complex, taxi instructions. Each structured route was presented on a single sheet of paper, as in the example presented in Figure 1 (left panel). Ground controllers received these structured routes as graphical images with the name of the taxi route clearly labeled across the card, as shown in the right panel of Figure 1.

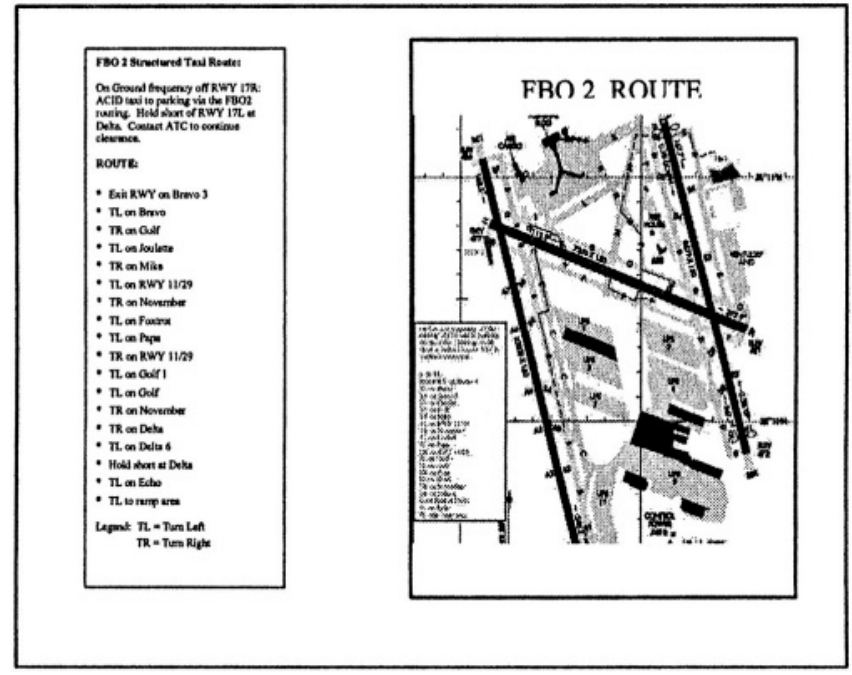

Figure 1. An Example of a Structured Inbound Taxi Route to Fixed Base Operations (FBO)

\subsubsection{Traditional Unstructured Taxi Routes}

A majority of the airport surface operations were performed using established taxi patterns, procedures and operations to and from the assigned runway and designated parking areas. For these unstructured, typical taxi routes, the ground controllers verbally provided pilots with the instructions necessary to taxi their aircraft to or from the assigned runways. Pilots did not know in advance the taxi routes they would be given.

\subsubsection{Digitized Audiotapes}

The Terminal Radar Approach Control (TRACON) facility provided one digital audiotape (DAT) for each test period. Separate voice records of all the transmissions made to the Ground East position were on the left channel. The right channel contained the Universal Time Coordinated (UTC) time code expressed in date, hour (hr), minute (min), and whole second (s). 


\subsection{Procedure}

During the operational evaluation, the tower was divided into two sections, with the West portion of the airspace dedicated to the evaluation. In addition, a portion of the airfield was set apart from normal operations and tower controllers limited access to the West runway to participating aircraft. Flight periods lasted between $2 \mathrm{hr} 19 \mathrm{~min}$ and $2 \mathrm{hr} 59 \mathrm{~min}$. The experimental flight periods were scheduled during normally low airport activity.

A majority of the airport surface operations were performed using customary taxi procedures ${ }^{15}$ with the unstructured taxi routes - following initial call-up ground controllers issued taxi clearances such as the one in FAA Order 7710.65M Air Traffic Control (2000): “American Four Ninety Two, Runway Three Six Left, taxi via taxiway Charlie, hold short of Runway Two Seven Right." However, for some portions of the taxi route, they instructed pilots to proceed according to the script-defined taxi routes - using the structured taxi-route cards. The distance, the numbers of turns, and the complexity of the inbound and outbound taxi routes were controlled.

Prior to each flight period, ground controllers were instructed to clear participating aircraft via customary taxi routes (i.e., unstructured taxi routes) or defined structured taxi routes and monitor the aircraft's movement along its assigned taxi route to ensure compliance with the scripted scenario and FAA procedures. During pre-flight briefings pilots received sets of taxi-route cards to use when issued structured taxi-route clearances. They were instructed to interpret the textual route information presented on their taxiroute cards to determine the route to taxi.

Pilots taxied their aircraft along their assigned routes using Paper-Charts (35 segments), Track-up (11 segments) or North-up (22 segments) surface map overlays to find their way to the runway, ramp, or transient parking area. Each outbound taxi segment lasted between $530.0 \mathrm{~s}$ and $1763.0 \mathrm{~s}$ $(\mathrm{M}=1289.0 \mathrm{~s}, \mathrm{SE}=89.11 \mathrm{~s})$ during 9 structured routes and from $292.0 \mathrm{~s}$ to $1652.0 \mathrm{~s}(\mathrm{M}=717.4 \mathrm{~s}, \mathrm{SE}=59.8 \mathrm{~s})$ during 20 unstructured routes. Each inbound taxi segment lasted between $520.0 \mathrm{~s}$ and $1321.0 \mathrm{~s}(\mathrm{M}=734.82 \mathrm{~s}$, $\mathrm{SE}=57.0 \mathrm{~s})$ for 22 structured routes and from $134.0 \mathrm{~s}$ to $470.0 \mathrm{~s}(\mathrm{M}=280.1 \mathrm{~s}$, $\mathrm{SE}=64.8 \mathrm{~s}$ ) for 17 unstructured routes.

15 FAA Order 7110M Air Traffic Control, 3-7-2. TAXI AND GROUND MOVEMENT OPERATIONS was current at the time of the evaluation. "Issue, as required or requested, the route for the aircraft/vehicle to follow on the movement area in concise and easy to understand terms. When a taxi clearance to a runway is issued to an aircraft, confirm the aircraft has the correct runway assignment." 


\subsection{Experimental Design}

This study used a two-factor, between-groups design. The betweengroups factors were Taxi Route (Structured, Unstructured) and Type of Surface Map (Paper-Chart, North-up, and Track-up). Each structured and unstructured taxi route segment was assigned to a different, pre-selected flight-crew as part of their outbound or inbound taxi segment.

The type of ADS-B equipment installed in each aircraft determined its assignment to a Type of Map group. Nine aircraft comprised the Paper-Chart Group. They could display ADS-B equipped aircraft on their CDTI, but no map overlay was available of the airport surface. Five of the aircraft had scanned Jeppesen airport surface map overlays on their CDTI, always depicted in a north-up orientation. They were classified as the North-up Group. The remaining two aircraft were classified as the track-up group, since their aircraft had a CDTI with a vector-based moving map of the airport surface map available for display. The messages recorded during the structured and unstructured routes allowed for a comparison with taxi performance by the Paper-Chart, North-up, and Track-up Groups.

\subsection{Dependent Measures}

Operational efficiency for each structured and unstructured taxi segment was of primary interest. It consisted of communication workload and operational communications. Measures of communication workload included number and duration of communication. Measures of operational communication included problems and operational concerns.

To measure changes in communication workload and operational communication, the messages transmitted between the ground controller and pilot of each aircraft were grouped into transactional communication sets (TCSs) that included the pilot's first message to the ground controller and the last message that either switched the pilot to local control (outbound) or terminated at the ramp or transient parking area (inbound). TCSs are made up of communication sets that comprise all the messages between a controller and pilot that share a common goal or purpose (Prinzo 1996). 


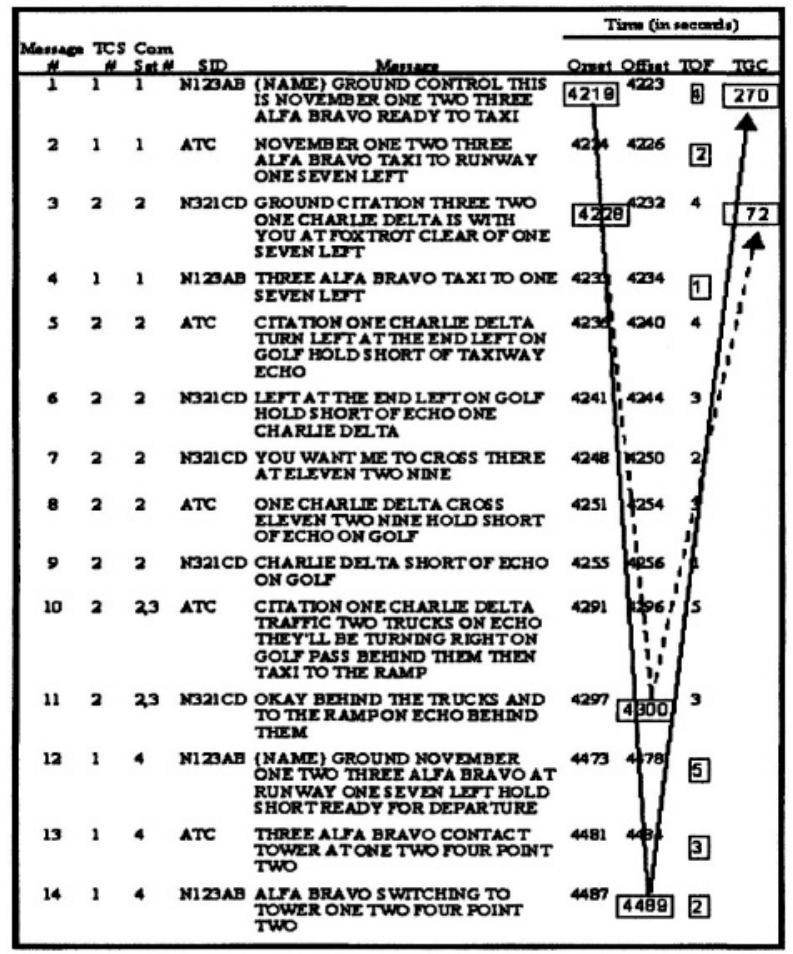

Figure 2. Example of a Transcript Encoded into Transactional Communication Sets

To illustrate, consider the partially encoded transcript presented in Figure 2. There are two TCS, one for each of two different taxi operations. TCS \#1, which is an outbound taxi, consists of two communication sets: a taxi route clearance (messages 1,2, and 4) and transfer of communications (messages 12 through 14). TCS \#2 is comprised of three communication sets: position report (message 3), taxi route clearance (messages 5 through 11), traffic advisory (messages 10 and 11). Message 10 is complex, in that the first part of the message is a traffic-advisory while the latter part is a taxi instruction.

\subsubsection{Objective Measures of Communication Workload}

Four measures of communication workload were examined for each TSC. They included (1) number of messages transmitted, (2) time on frequency per message (TOF), (3) frequency occupancy time (FOT), and (4) time under ground control (TGC).

As shown in Figure 2, six messages involved N123AB (TCS \#1). The TOF for the first message was $4 \mathrm{~s}$. Frequency occupancy tune for TCS \#1 was computed as the sum of the TOF. In the example, FOT was $17 \mathrm{~s}$ (FOT $=$ 
$\Sigma$ TOF $=4+2+1+5+3+2)$. As illustrated by the solid-line arrow, the time $\mathrm{N} 123 \mathrm{AB}$ spent under ground control (time ground control, TGC) was computed as the time lapsed from the onset of the pilot's initial call-up in message 1 (at $4219 \mathrm{~s}$ ) to the closing of the transaction in message 14 (at 4489 s). In the example, TGC was 270 s (4489 s - 4219 s). N321CD's TGC is shown by the dashed-line arrow and it was $72 \mathrm{~s}$. A taxi segment typically began with a pilot checking in and ended in the transfer of communication to local control (outbound route), as was the case with TCS \#1, or with the last recorded transmission as the pilot navigated back to either the ramp or transient parking area (inbound), which was the case with TCS \#2.

\subsubsection{Measures of Operational Communications}

Communications that have the potential to adversely affect operational efficiency were identified and classified as problems and operational concerns. Problems included message reception (say again, did you copy), misunderstanding (readback error, stolen transmission, intentional repetition of a previous message for emphasis), erroneous information (incorrect call sign, can't find route segment provided in taxi instructions), and message production (self-correction of the call sign or another piece of information). Operational concerns involved spatial and positional awareness. Spatial awareness includes a general understanding of the airport's surface geography (aircraft is not on its assigned route, correction made to a previous taxi instruction, incorrect taxi clearance issued, instructions given to rejoin route, confusion, lost, missed turn), whereas positional awareness concerns the temporal and relational factors associated with maneuvering about the airport (maneuver around aircraft, possible conflict, request clearance to cross an active runway).

\section{RESULTS}

Operational communications were evaluated from verbatim transcripts and digitized voice recordings provided by the TRACON facility. Although requests were made during the planning of the event that baseline circuits be included, none were conducted. The analysis of voice tapes did allow for preliminary comparisons between flight crews who had access to traditional paper-charts and electronic airport surface maps, in either a north-up or track-up orientation, as aids to their surface situational awareness.

The analyses were restricted to taxi routes that either began or ended at the ramp or transient parking areas. Since progressive ground movement instructions include step-by-step routing directions, these taxi routes were 
excluded because pilot variance in navigating on the airport surface would be restricted as ground control would be providing detailed instructions to guarantee safe and expeditious flow to the destination point. There were 727 messages (pilots $=401$, controllers $=326$ ) transmitted between participating pilots and controllers during the 31 structured and 37 unstructured taxi routes that involved 39 inbound and 29 outbound taxi segments.

\subsection{Communication Workload}

Multivariate Analysis of Variance (MANOVA) was performed on the taxi-segment means for each objective measure of communication workload presented in Table 1 (standard errors (SE) are enclosed in parentheses). Univariate Analysis of Variance (ANOVA) was used to assess the statistically significant findings. The Tukey Honestly Significant Difference (HSD) statistic was performed on statistically significant main effects and interactions. An alpha level of .05 was set for all statistical tests.

Table 1. Objective Measures of Communication Workload

\begin{tabular}{lcccr}
\hline & \multicolumn{4}{c}{ Measures of Communication Workload, in seconds (Standard Error) } \\
\cline { 2 - 5 } & TOF & N Nessages & \multicolumn{1}{l}{ FOT } & \multicolumn{1}{l}{ TGC } \\
\hline Structured Taxi Route & & & \\
Paper-chart & $3.24(.25)$ & $11.00(1.13)$ & $35.89(3.69)$ & $716.67(63.88)$ \\
North-up & $3.51(.37)$ & $16.50(1.70)$ & $52.63(5.54)$ & $1383.88(95.81)$ \\
Track-up & $3.59(.47)$ & $10.60(2.14)$ & $35.20(7.00)$ & $759.20(121.19)$ \\
Unstructured Taxi Route & & & \\
Paper-chart & $3.64(.25)$ & $11.24(1.16)$ & $36.82(3.80)$ & $688.24(65.73)$ \\
North-up & $3.67(.28)$ & $7.71(1.28)$ & $27.86(4.18)$ & $299.36(72.43)$ \\
Track-up & $4.28(.43)$ & $7.50(1.96)$ & $32.33(6.39)$ & $536.50(110.63)$ \\
\hline
\end{tabular}

A two-way Type-of-Route by Type-of-Map MANOVA revealed a statistically significant main effect for Type of Route $[\mathrm{F}(4,59)=9.76]$ and Type-of-Route by Type-of-Map interaction, $[\mathrm{F}(8,118)=6.85]$. Subsequent Univariate ANOVAs revealed that not only were more messages transmitted during structured $(\mathrm{M}=12.70 \mathrm{SE}=.99)$ compared with unstructured $(\mathrm{M}=8.82$ $\mathrm{SE}=.87)$ taxi routes $[\mathrm{F}(1,62)=8.72]$ but more time was spent under the authority of ground control (Structured $\mathrm{M}=953.25 \mathrm{SE}=55.73$, Unstructured $\mathrm{M}=508.03 \mathrm{SE}=49.22)[\mathrm{F}(1,62)=35.86]$. Although the time on frequency to transmit individual messages did not vary with the type of route navigated $[F(1,62)=2.09]$, the overall frequency occupancy time increased by $9 \mathrm{~s}$ during the structured $(\mathrm{M}=41.24 \quad \mathrm{SE}=3.22)$ compared with unstructured $(\mathrm{M}=32.34 \mathrm{SE}=2.84)$ taxi routes, $[\mathrm{F}(1,62)=4.29]$.

The statistically significant interaction revealed that the type of route navigated, in combination with the type of map available on the flight deck, affected communication workload for the number of messages transmitted 
$[F(2,62)=5.70]$, frequency occupancy time $[F(2,62)=4.48]$ and time spent under the authority of ground control $[\mathrm{F}(2,62)=25.02]$. In particular, Tukey post hoc comparisons clearly indicated that controllers and pilots in the north-up surface map group exchanged twice as many messages during the structured routes, as compared with unstructured routes.

The north-up surface map group spent nearly twice as long communicating during the structured, as opposed to unstructured taxi routes, and was under the authority of ground control for an additional 18 mins. Tukey results also showed that when the north-up surface map group traveled via structured taxi routes they exchanged more messages than the track-up surface map group that navigated the unstructured taxi routes. They also spent more time on frequency than the track-up surface map group during structured taxis and spent more time under the authority of ground control than the other participating groups, regardless of their assigned type of taxi route. The north-up surface map group that navigated unstructured taxi routes spent the least time under the authority of ground control.

\subsection{Operational Communication}

Previous research has demonstrated that when attentional resources are taxed, people may fail to detect that another person is talking, they may misspeak or mishear, or experience other problems identified from communication (Navarro, 1989). To gain some insights as to how the combination of the type of route navigated and type of map available affected communication workload, an examination of the operational communications was initiated.

\subsubsection{Types of Problems}

As shown in Table 2, 40\% of the problems involved message reception (request from a pilot to have a transmission repeated, controller request for confirmation that a message was received). Another $16 \%$ of the problems involved misunderstandings (pilots incorrectly repeating back information or responding to an instruction meant for someone else). Erroneous information (5.2\% of the problems) involved an incorrectly spoken aircraft call sign and pilot failure to find a taxi intersection. Mid-streamcorrections, consisted of mid-utterance repairs that involved either the aircraft's call sign or taxi information, and they included $34.2 \%$ of the identified problems. Lastly, intentional repetition or restatement of an earlier transmission occurred 5.3\% of the time. In each recurrence, the controllers instructed the pilots to navigate their aircraft along the assigned taxi routes or runways. 


\begin{tabular}{lllllllll}
\hline & \multicolumn{6}{c}{ Type of Map } & \\
\cline { 2 - 7 } & \multicolumn{2}{c}{ Paper-chart } & \multicolumn{2}{c}{ Track-up } & \multicolumn{2}{c}{ North-up } & \\
\cline { 2 - 7 } $\begin{array}{l}\text { Types of Problem } \\
\text { (n=38) }\end{array}$ & $\mathrm{S}$ & $\mathrm{U}$ & $\mathrm{S}$ & $\mathrm{U}$ & $\mathrm{S}$ & $\mathrm{U}$ & Total \% \\
\hline Message Reception & $5.3 \%$ & $10.6 \%$ & $5.3 \%$ & $2.6 \%$ & $7.9 \%$ & $7.9 \%$ & $39.4 \%$ \\
Misunderstanding & $5.3 \%$ & $5.3 \%$ & & & $2.6 \%$ & $2.6 \%$ & $15.8 \%$ \\
Erroneous Information & $2.6 \%$ & $2.6 \%$ & & & & & $5.3 \%$ \\
Message Production & $5.3 \%$ & $10.6 \%$ & $2.6 \%$ & $2.6 \%$ & $13.1 \%$ & & $34.2 \%$ \\
Intentional Repetition & $2.6 \%$ & $2.6 \%$ & & & & & $5.3 \%$ \\
Total \% & $21.1 \%$ & $31.6 \%$ & $7.9 \%$ & $5.3 \%$ & $27.3 \%$ & $10.5 \%$ & $100.0 \%$ \\
\hline
\end{tabular}

\subsubsection{Types of Operational Concerns}

The types of operational concerns, along with their frequency of occurrence, are presented in Table 3. The operational concerns noted in the data involved either spatial awareness (aircraft is not on its assigned route, correction to taxi instructions, incorrect taxi clearance issued, instructions given to rejoin route, lost, missed turn) or positional awareness (maneuver around aircraft, possible conflict, request cleared to cross a runway). Approximately $75 \%$ of the operational concerns were related to spatial awareness, while the remaining $25 \%$ centered on position awareness.

\subsection{Prevalence of Problems and Operational Concerns}

Before examining the data for problems and operational concerns, a chisquare $\left(\chi^{2}\right)$ test revealed no statistically significant difference in the number of structured, compared with unstructured taxi routes completed, when pilots had available either the paper-chart, track-up or north-up surface maps, $\left[\chi^{2}(2)=1.24\right]$. Of the 68 taxi routes, 37 contained one or more problem. Subsequent chi-square tests revealed a significant difference in the number of problematic routes among participants in the north-up surface map group $\left[\chi^{2}(1)=12.57\right]-100 \%$ of their structured $(8 / 8)$ and $21 \%$ of their unstructured (3/14) taxi routes were problematic. The number of problematic routes was equivalent when pilots navigated their assigned structured or unstructured taxi routes with paper-charts (Structured=11/18 Unstructured=10/17) or track-up (Structured=3/5 Unstructured=2/6) surfacemap displays.

Table 3. Frequency of Operational Concerns Presented Type of Route by Type of Map

Types of Operational

\begin{tabular}{|c|c|c|}
\hline \multicolumn{3}{|c|}{ Type of Map } \\
\hline Paper-chart & Track-up & North-up \\
\hline $\mathrm{U}$ & $\mathbf{U}$ & $\mathrm{U}$ \\
\hline
\end{tabular}

Concerns ( $\mathrm{n}=33$ ) 


\begin{tabular}{|c|c|c|c|c|c|c|c|}
\hline \multirow{2}{*}{ assigned route } & \multicolumn{6}{|c|}{ Type of Map } & \multirow[b]{3}{*}{$6.1 \%$} \\
\hline & & & & & & & \\
\hline $\begin{array}{l}\text { Corrected taxi } \\
\text { instructions }\end{array}$ & & & $3.0 \%$ & & $3.0 \%$ & & \\
\hline Incorrect taxi clearance & & & & & & $6.1 \%$ & $6.1 \%$ \\
\hline $\begin{array}{l}\text { Instructions given to } \\
\text { rejoin route }\end{array}$ & $3.0 \%$ & & & & & $6.1 \%$ & $9.1 \%$ \\
\hline Lost & $6.1 \%$ & & & & & & $6.1 \%$ \\
\hline $\begin{array}{l}\text { Missed turn } \\
\text { Position Awareness }\end{array}$ & $3.0 \%$ & & $3.0 \%$ & & $6.1 \%$ & & $12.1 \%$ \\
\hline $\begin{array}{l}\text { Position Awareness } \\
\text { Maneuver around } \\
\text { aircraft }\end{array}$ & & $3.0 \%$ & & & & & $3.0 \%$ \\
\hline Possible conflict & $3.0 \%$ & $3.0 \%$ & & $3.0 \%$ & & $3.0 \%$ & $12.1 \%$ \\
\hline $\begin{array}{l}\text { Request cleared to cross } \\
\text { a runway }\end{array}$ & $15.2 \%$ & $9.1 \%$ & $3.0 \%$ & & $3.0 \%$ & $3.0 \%$ & $33.3 \%$ \\
\hline Total & $33.3 \%$ & $15.1 \%$ & $15.0 \%$ & $3.0 \%$ & $27.3 \%$ & $6.0 \%$ & $100.0 \%$ \\
\hline
\end{tabular}

A two-way Type-of-Route by Type-of-Map ANOVA was performed on the total number of problems and operational concerns associated with each assigned taxi-route clearance. As shown in Figure 3, more problems and operational concerns were present during the structured, as compared with the unstructured taxi routes $[\mathrm{F}(1,62)=9.82]$.

The Type-of-Route by Type-of-Map interaction also was statistically significant $[F(2,62)=3.90]$. Subsequently, the Tukey HSD statistic revealed significantly more overall problems; operational concerns resulted only for the north-up surface-map group during structured taxi routes, compared with the north-up and track-up surface map groups during unstructured taxi routes. Five of the 9 identified problems occurred during eight taxi operations and involved mid-stream corrections, three centered on problems in message reception, and one involved an incorrect readback of a taxi clearance. Eight of the 9 identified operational concerns involved spatial awareness (missed turn $=2$, aircraft not on its assigned route $=1$, ATC issued instructions to rejoin a route $=2$, incorrect taxi clearance issued $=2$, and correction to taxi instructions $=1$ ), and one concerned position operation (e.g., a pilot request to cross an active runway). 


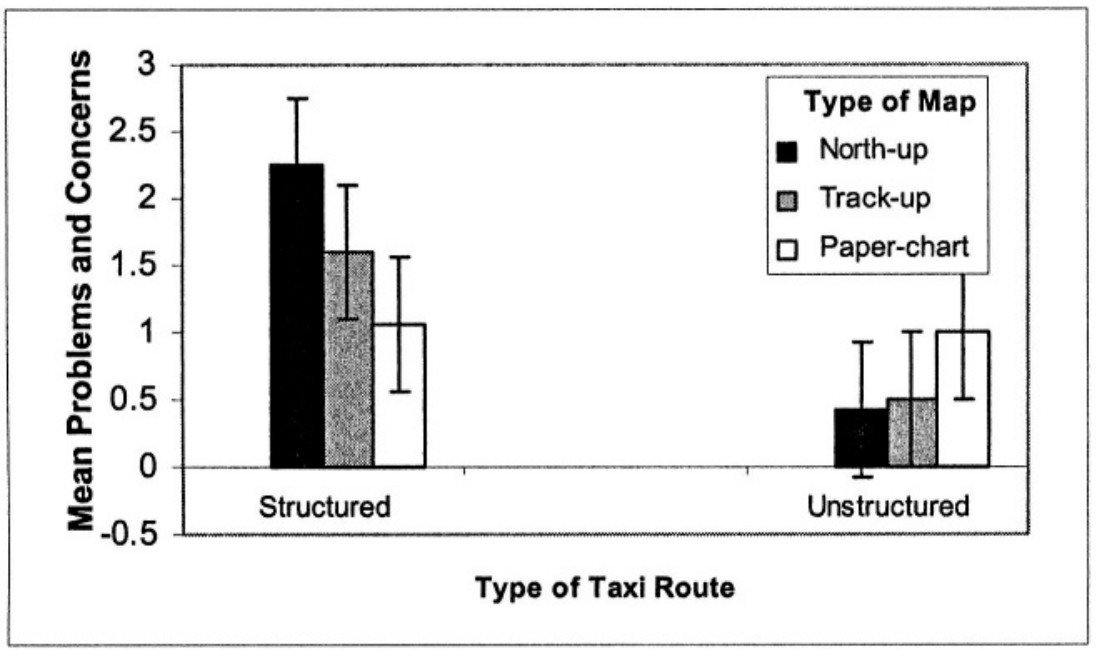

Figure 3. Problems and Operational Concerns Presented by Type of Route and Type of Map

\section{DISCUSSION}

The analysis of voice communications revealed that the combination of the type of assigned taxi route and surface map capability onboard the aircraft affected communication workload. Notably, participants in the north-up surface map group a) exchanged more messages than the track-up surface map group when they navigated the unstructured taxi routes, b) spent more time on frequency than the track-up surface map group during the structured taxi condition, and c) spent more time under the authority of ground control than the other groups regardless of their assigned taxi route (structured or unstructured).

Of the 68 taxi routes completed, 54\% contained one or more operational concern or problem - with more overall problems in the north-up surface map group during the structured taxi routes, when compared with the northup and track-up surface map groups during unstructured taxi routes. Notably, all of the structured and $21 \%$ of the unstructured taxi routes that involved the use of a north-up display were problematic. The number of problematic routes was equivalent when pilots navigated their assigned structured or unstructured taxi routes using paper-charts or track-up surface-map displays.

The results of the Wickens, Liang, Prevett, and Olmos (1996) simulation study, which investigated pilot use of either a rotating or fixed map display, reported that access to a north-up display was not advantageous for pilots flying southerly headings - any changes to their flight path would require complex mental rotations. Their interpretation correlates well to the findings 
reported here in that about $75 \%$ of the identified operational concerns involved spatial awareness. The north-up map group experienced more problems that related to missing a turn or attempting to rejoin a taxi route while problems for the paper-chart group took the form of getting lost; for the track-up map group, it was not being on their assigned route.

It would seem that having a north-up map display for airport surface navigation provided no additional benefit over a paper chart. Furthermore, pilots in the paper-chart and north-up map groups may have been busier performing complex mental operations (i.e., making left to right transformations) while navigating. Pilots in these map groups requested more repetitions and were more likely to incorrectly read back messages. Consequently, fewer attentional resources may have been available to actively listen for their aircrafts' call signs.

This is similar to driving on an unfamiliar metropolitan interstate highway requiring extra vigilance to make a timely and correct turn when approaching a fast-moving, cloverleaf intersection. If a passenger should attempt to engage the driver in casual conversation, the driver might miss the turn or not hear the passenger. The driver and pilot alike can request a "say again" or ask for assistance - both requiring additional communications.

Although some pilots (like drivers) turn their maps to be congruent with the direction they are going, Joseph et al. (2002) did not mention whether the pilots in the paper-chart group had rotated their maps or not. If they had, their performance should have been more aligned with the pilots in the trackup group instead of the north-up group.

When evaluating emerging avionics devices that aid navigation, consideration as to the format of these displays must be deliberated in light of the piloting task the operator is expected to perform (see Aretz, 1991, for a summary of previous research; Carel, McGarth, Hersherber, \& Herman, 1974, for early research on design criteria). The format in which a map is presented can, and does, affect some aspects of pilot performance - northup displays are better for some tasks (planning), and track-up displays are better at others (turning). In fact, Clarke, McCauley, Sharkey, Dingus, and Lee (1996) suggest that, when both north-up and track-up displays are available, pilots typically select north-up map displays when planning routes and track-up display when flying. Some developers are making both northup and track-up modes available on some of their CDTI devices, and this provides the pilot with the option to select one mode for some piloting tasks and the other mode for others. Of course, some pilots still may choose to use paper charts as their primary source of airport information. 


\section{REFERENCE}

Aretz, A. (1991). The design of electronic map displays. Human Factors, 33:85-101.

Carel, W.L., McGarth, J.J., Hershberger, M.L. and Herman, J.A. (1974). (DOD-74-731101). Design criteria for airborne map displays volume I: Methodology and research results. Washington DC: Department of Defense, Department of the Navy, Office of Naval Research.

Clarke, D.L., McCauley, M.E., Dingus, T.A., and Lee, J.D. (1996). Development of human factors guidelines for advanced traveler information systems and commercial vehicle operations: Comparable systems analysis. Washington, DC: Federal Highway Administration (FHWA-RD-95-197).

Federal Aviation Administration. (November 2002). Order 7050.1 Runwa Safety Program; http://www.awp.faa.gov.

Federal Aviation Administration. (July 2002). Runwa Safety Blueprint 2002-2004; http://www.faa.gov/runwaysafety/publications.cfm.

Federal Aviation Administration. (2001). Operational Evaluation-2 Final Report; http://www1.faa.gov/And/AND500/docmgr/docs/T0247.pdf.

Federal Aviation Administration (2000). Air Traffic Control (7110.65M). Washington DC: U.S. Government Printing Office.

Federal Aviation Administration (1998). Airport Surface Operations Safety Action Plan; http://www1.faa.gov/ats/ato/150_docs/RSP_AP.DOC.

Joseph, K.M., Domino, D. Battiste, V., Bone, R., and Olmos, O. (2003). A summary of flightdeck observer data from SafeFlight 21 OpEval-2. (DOT/FAA/AM-03/2). Washington, DC: Federal Aviation Administration, Office of Aerospace Medicine.

National Transportation Safety Board. (July 2000). NTSB Safety Recommendation A-00-66; http://www.ntsb.gov/Recs/letters.

National Transportation Safety Board. (1986). Runway Incursions at Controlled Airports in the United States. NTIS Report PB86-917003.

Prinzo, O.V. (1996). An analysis of approach control/pilot voice communications. (DOT/FAA/AM-96/26). Washington, DC: Federal Aviation Administration, Office of Aerospace Medicine.

Wickens, C.D., Liang, C., Prevett, T., and Olmos, O. (1996). Electronic maps for terminal area navigation: Effects of frame of reference and dimensionality. The International Journal of Aviation Psychology, 6: 241-71. 